\title{
Evaluation of Low-Dose Ketamine on Inflammatory Biomarker Profile Following Off-Pump Coronary Artery Bypass Grafting
}

\author{
Daljeet Singh ${ }^{1}$ Ramesh Kashav ${ }^{2}$ Rohan Magoon ${ }^{2}$ \\ Aastha Gupta ${ }^{1}$ Vijay Gupta ${ }^{3}$ \\ ${ }^{1}$ Department of Anaesthesia, Atal Bihari Vajpayee Institute of \\ Medical Sciences (ABVIMS) and Dr Ram Manohar Lohia Hospital, \\ New Delhi, India \\ 2Department of Cardiac Anaesthesia, Atal Bihari Vajpayee Institute \\ of Medical Sciences (ABVIMS) and Dr Ram Manohar Lohia Hospital, \\ New Delhi, India \\ ${ }^{3}$ Department of Cardiothoracic and Vascular Surgery, Atal Bihari \\ Vajpayee Institute of Medical Sciences (ABVIMS) and Dr Ram \\ Manohar Lohia Hospital, New Delhi, India
}

Jasvinder Kaur Kohli² Mohandeep Kaur ${ }^{1}$

J Card Crit Care:2020;4:33-39

\begin{abstract}
Address for correspondence Ramesh Kashav, MD, Department of Cardiac Anaesthesia, Atal Bihari Vajpayee Institute of Medical Sciences (ABVIMS) and Dr Ram Manohar Lohia Hospital, Baba Kharak Singh Marg, New Delhi 110001, India (e-mail: drkashav@yahoo.co.in).
\end{abstract}

\begin{abstract}
Keywords

- inflammatory markers

- Ketamine

- OPCAB

The role of ketamine in attenuating the postoperative inflammatory response is a subject of recent clinical interest. Despite several studies elucidating the anti-inflammatory potential of ketamine in on-pump coronary artery bypass grafting and noncardiac surgeries, the literature on the role of ketamine in ameliorating systemic inflammatory response syndrome following off-pump coronary artery bypass grafting (OPCAB) is rather scarce. This prospective observational study was designed to compare the postoperative inflammatory milieu with and without the addition of low dose $(0.5 \mathrm{mg} / \mathrm{kg})$ of ketamine to the anesthetic regimen in the patients undergoing OPCAB, wherein 60 patients were allocated to group T, receiving ketamine $(n=30)$ and the group $C$, not receiving ketamine $(n=30)$. The estimated biomarkers included $C$-reactive protein (CRP), interleukin-6 (IL-6), tumor necrosis factor- $\alpha(T N F-\alpha)$, troponin $T$, and creatine kinase-MB (CK-MB) at the following time points: prior to induction (T0), immediate postoperative period (T1), 4 hours postoperatively (T2), 24 hours postoperatively (T3), and 48 hours postoperatively (T4). The major finding of the study was a significant reduction in the postoperative values of CRP and IL- 6 at T1, T2, T3, and T4 $(p<0.001)$ However, postoperative serum levels of TNF- $\alpha$, CK-MB, and troponin T were comparable in the group $T$ and group C. The average postoperative CRP and IL- 6 levels positively correlated with the VISh (the highest postoperative vasopressor inotrope score) with the $r$ values of $0.733(p<0.001)$ and $0.704(p<0.001)$, respectively. The index study substantiates the anti-inflammatory potential of ketamine in preventing immune function alterations attributable to anesthesia and cardiac surgery.
\end{abstract}

\section{Introduction}

The burden of atherosclerotic disease continues to escalate with a heightened number of comorbid patients presenting with coronary artery disease. This emanates largely as a combination of an increased life expectancy and improved diagnostic modalities. Albeit, the development of the spectrum of interventions employed to re-perfuse the ischemic myocardium, like thrombolysis, percutaneous coronary angioplasty, and surgical revascularization continues to be the mainstay of treatment for the patient cohort with multivessel disease and complex anatomy. ${ }^{1,2}$
DOI https://doi.org/

10.1055/s-0040-1713299 ISSN 2457-0206.
License terms

() (1) $\Theta \circledast$ 
Conventionally, systemic inflammatory response syndrome (SIRS) is inexorably linked to the conduct of cardiac surgery employing cardiopulmonary bypass ( $\mathrm{CPB}$ ) which detrimentally affects the postoperative outcomes. ${ }^{3-5}$ Therefore, an increasing number of surgical centers are contemplating beating heart surgical revascularization. However, an off-pump coronary artery bypass grafting (OPCAB) also incurs varying degree of inflammatory response activation which is accentuated by the accompanying myocardial ischemia-reperfusion injury (IRI). ${ }^{6,7}$ This reduced but yet not completely obliterated inflammatory response continues to influence postoperative cardiac function and clinical outcomes following OPCAB.

Various anti-inflammatory strategies and pharmacological agents have been evaluated in the cardiac surgical arena over the last decade. These include (not limited to) leukocyte filtration, corticosteroids, aprotinin, and nitric-oxide donor compounds. ${ }^{8-11}$ Considering a favorable cost-benefit ratio and an acceptable adverse effect profile of anti-inflammatory modalities, several anesthetic agents have been proposed to demonstrate a protective role against IRI and subsequent SIRS. The putative mechanisms of protection include: attenuation of $\mathrm{Ca}^{2+}$ overload, anti-inflammatory and antioxidant effects, pre- and postconditioning effects.

$\mathrm{N}$-methyl-D-aspartate (NMDA) receptor antagonist, ketamine has demonstrated anti-inflammatory role in recent studies ${ }^{12-15}$ by decreasing the serum levels of proinflammatory markers, such as C-reactive protein (CRP) and interleukin-6 (IL-6) following CPB..$^{16,17}$ Amelioration of SIRS with ketamine is thought to be mediated through inhibition of nuclear factor (NF)-kB, ${ }^{7}$ which regulates the transcription of genes that encode the production of proinflammatory cytokines. ${ }^{18,19}$ This positive modulation confers an anti-inflammatory potential to the drug by limiting the exacerbation of systemic inflammation with minimal effect on the local healing processes.

Therefore, we evaluated whether the low dose of ketamine administered during induction of anesthesia would reduce the activity of proinflammatory mediators in patients undergoing $\mathrm{OPCAB}$ in a prospective, randomized double-blind study.

\section{Materials and Methods}

After obtaining approval from the Institutional Review Board and Institutional Ethics Committee, this prospective observational study was conducted at our tertiary cardiac center from November, 2015 to March, 2017. Sixty patients posted for elective OPCAB were allocated to either control (C group, not receiving ketamine) or test (T group, receiving ketamine) by computer-based randomization. Patients were excluded from the study if they met one of the following criteria: recent ( $<14$ days) myocardial infarction, unstable angina with elevated creatine kinase-MB (CK-MB) elevated serum creatinine $(>1.3 \mathrm{mg} / \mathrm{dL}$ ) before surgery, previous cardiac surgery, previous stroke or pulmonary disease, history of treatment with steroid or nonsteroidal anti-inflammatory drugs within a month, patients who are on vasoactive medications, patients undergoing re-exploration surgery or ejection fraction less than $40 \%$, and intraoperative hemodynamic instability necessitating conversion to on pump.

A thorough preoperative evaluation was performed, and a written informed consent was obtained from all the patients. The included subjects were fasted preoperatively as per The American Society of Anesthesiologists guidelines and received tablet alprazolam $0.25 \mathrm{mg}$ and ranitidine $150 \mathrm{mg}$ orally, the night before surgery and on the morning of surgery. After wheeling in the patient into the operating room, pulse oximeter (SpO2) and five lead electrocardiogram electrodes were applied and patient's noninvasive vital parameters were recorded which include heart rate (HR), systolic and diastolic blood pressures (BPs), and mean arterial pressure (MAP). After local anesthetic infiltration and under all aseptic precautions, a wide bore intravenous cannula and a 20-G intra-arterial right radial artery catheter were secured. Anesthesia was induced with propofol $(2 \mathrm{mg} / \mathrm{kg})$ and bolus of ketamine $1 \%(0.5 \mathrm{mg} / \mathrm{kg})$ in the test group or propofol (2 $\mathrm{mg} / \mathrm{kg}$ ) and same volume of normal saline (as ketamine) in the control group, according to randomization. Apart from the induction agent, rest of the anesthesia technique was similar in both the groups as per the institutional protocol.

After induction a Swan-Ganz catheter (Edwards Life Sciences, Irwine, California, United States) was positioned in the pulmonary artery through 7-Fr sheath inserted in the right internal jugular vein. The hemodynamic parameters in the form of HR, BP, central venous pressure (CVP), pulmonary capillary wedge pressure (PCWP), cardiac output (CO) (by FloTrac continuous cardiac output monitor), cardiac index (CI), systemic vascular resistance and systemic vascular resistance index (SVR and SVRI), and pulmonary vascular resistance and pulmonary vascular resistance index (PVR and PVRI) were monitored continuously. Surgical revascularization was contemplated in the background of activated clotting time value of more than 300 seconds following administration of heparin in the dosage of $1 \mathrm{mg} / \mathrm{kg}$. The intraoperative hemodynamic parameters were titrated within $20 \%$ of the baseline values with the initiation of vasopressor agents (if, SVRI $<1,500 \mathrm{dyne} / \mathrm{s} / \mathrm{cm}^{5} / \mathrm{m}^{2}$ ) or inotropic agents (if $\mathrm{Cl}<2.5 \mathrm{~L} / \mathrm{min} / \mathrm{m}^{2}$ ), to maintain MAP of more than $60 \mathrm{~mm} \mathrm{Hg}$. Intravascular volume replacement was managed with crystalloid and colloid solutions to maintain PCWP between 8 and $16 \mathrm{~mm} \mathrm{Hg}$ according to the baseline values. The hemodynamic parameters in the form of HR, BP, CVP, PCWP, CO (by FloTrac/Vigileo continuous cardiac output monitor), CI, SVR and SVRI, and PVR and PVRI were monitored continuously. Heparin was reversed with protamine in dose of 1.3 times the dose of heparin administered. All patients were transferred to the intensive care unit after surgery.

\section{Biochemical Marker Analysis}

Arterial blood samples for the measurement of serum levels of CRP, interleukin-6 (IL-6), tumor necrosis factor- $\alpha$ (TNF- $\alpha$ ), troponin $\mathrm{T}(\mathrm{TnT})$, and CK-MB were drawn just prior to induction (T0), immediate postoperative period (T1), at 4 hours postoperatively (T2), 24 hours postoperatively (T3), and 48 hours postoperatively (T4). The arterial blood samples 
drawn were centrifuged and serum levels of CRP and CK-MB were measured by dry chemistry analyzer (Vitros 350) on everyday basis in institutional laboratory. Centrifuged serum for IL-6, TNF- $\alpha$, and TnT was stored at $70^{\circ} \mathrm{C}$ and measured using enzyme-linked immunosorbent assay (ELISA) technique in batches employing human IL-6 ELISA kit (Sigma Aldrich, Catalogue no. RAB0306), human TNF- $\alpha$ ELISA kit (Sigma Aldrich, Catalogue no.RAB0476), and TnT (TNNT1 gene) human ELISA kit (Invitrogen, Catalogue no. EHTNNT1), respectively.

In addition to continuous monitoring during the preoperative (T0) and intraoperative period, hemodynamic parameters were also recorded in postoperative periods at $\mathrm{T} 1, \mathrm{~T} 2, \mathrm{~T} 3$ and $\mathrm{T} 4$.

\section{Statistical Analysis}

The sample size was estimated based on the results of previous-related studies ensuring an $80 \%$ power of the study. The statistical analysis was performed using the statistical package for the social science system version SPSS 21.0. The continuous variables were presented as mean $\pm S D$, and categorical variables were presented as absolute numbers and percentages. The normality of the data was tested by Kolmogorov-Smirnov test. If the normality was rejected, then nonparametric test was used. The comparison of normally distributed continuous variables between the groups was performed using Student's t-test/Mann-Whitney test (when the datasets were not normally distributed). Nominal categorical data between the groups were compared using Chi-square test or Fisher's exact test as appropriate. Value of $p<0.05$ was considered statistically significant. Spearman's rank correlation coefficient was used to identify correlations between nonparametric variables.

\section{Results}

A total of 60 patients completed the study. The demographic factors and surgical data were comparable in the two groups (-Table 1). There was no significant difference in the perioperative hemodynamic profile of the two groups ( - Table 2 ).

The inflammatory markers were compared between the two groups. All the biomarkers (IL-6, CRP, TNF- $\alpha$, CK-MB, and TnT) were comparable at T0. The serum CRP and IL-6 levels were significantly $(p<0.05)$ lower at all postoperative time points $(\mathrm{T} 1, \mathrm{~T} 2, \mathrm{~T} 3, \mathrm{~T} 4)$ in the ketamine group in contrast to the control group (- Table 3 ). However, the postoperative
TNF- $\alpha$, TnT, and CK-MB levels were comparable between the two groups. The time trend analysis of postoperative CRP and IL-6 levels is depicted in - Fig. 1, wherein both the groups demonstrate an increase in the inflammatory marker profile. It is noteworthy that the elevation in the inflammatory marker profile was higher in group $\mathrm{C}$ as compared with group $\mathrm{T}$. The postoperative outcomes were comparable between the two groups. However, it was noteworthy that the highest postoperative $\mathrm{VIS}_{\mathrm{h}}$ (vasopressor inotrope score in the first postoperative 48 hours) was significantly higher for the patients in control group as opposed to the test group (-Table 4). Interestingly, the average postoperative CRP and IL-6 levels positively correlated with the VIS $\mathrm{h}_{\mathrm{h}}$ with the $r$ values of $0.733(p<0.001)$ and $0.704(p<0.001)$, respectively (-Fig. 2).

\section{Discussion}

The major finding of the study is constituted by the demonstration of lower proinflammatory markers like CRP and IL-6 with addition of low-dose ketamine to the anesthetic induction regimen in the patients undergoing OPCAB. The statistically significant difference in the postoperative CRP and IL-6 levels persisted throughout the study period which lasted for the first postoperative 48 hours. Interestingly, these results are in contrast to a similar study by Cho et $\mathrm{al}^{20}$ who failed to demonstrate anti-inflammatory potential of ketamine in OPCAB setting. However, there are several studies elucidating the anti-inflammatory effect of ketamine in on-pump coronary artery bypass grafting (CABG) $)^{13-15}$ and noncardiac surgeries. ${ }^{12}$ Despite the reduction in CRP and IL-6, the index study outlined a comparable postoperative TNF- $\alpha$ levels in the ketamine group. At the same time there was no significant difference in the postoperative markers of the myocardial damage (TnT and CK-MB) in the two groups.

Ketamine, a noncompetitive NMDA receptor antagonist, in addition to its anesthetic activity possesses a positive immunomodulatory impact which can be of special interest in cardiac surgical patient. ${ }^{21,22}$ The anti-inflammatory effects of ketamine are mediated by inhibition of NF-kB, which regulates the transcription of genes that encode the production of proinflammatory cytokines. ${ }^{7}$ In most studies $^{13,14,16}$ anti-inflammatory effect of ketamine has been demonstrated mainly in terms of reducing the serum levels of proinflammatory mediators, such as CRP, IL-6, and TNF- $\alpha$.

Table 1 Demographic characteristics in two groups

\begin{tabular}{|l|l|l|l|}
\hline Parameters & Control $(\boldsymbol{n}=\mathbf{3 0})$ & Test $(\boldsymbol{n}=\mathbf{3 0})$ & $p$-Value \\
\hline Age $(\mathrm{y})$ & $60.27 \pm 9.45$ & $62.43 \pm 6.85$ & 0.359 \\
\hline Sex (female: male) & $8: 22$ & $8: 22$ & 1 \\
\hline BSA $\left(\mathrm{cm}^{2}\right)$ & $1.69 \pm 0.14$ & $1.67 \pm 0.16$ & 0.538 \\
\hline euroSCORE & $4.13 \pm 0.79$ & $4.02 \pm 0.83$ & 0.39 \\
\hline No. of grafts & $3.63 \pm 0.65$ & $3.48 \pm 0.73$ & 0.21 \\
\hline
\end{tabular}

Abbreviations: BSA, body surface area; euroSCORE, European System for Cardiac Operative Risk Evaluation.

Note: $p$-Value $<0.05$ is considered statistically significant. 
Table 2 Comparison of hemodynamic parameter between the control and ketamine group

\begin{tabular}{|c|c|c|c|c|}
\hline Parameters & Time & Control group $(n=30)$ & Test group $(n=30)$ & $p$-Value \\
\hline \multirow[t]{6}{*}{ MAP (mm Hg) } & T0 & $98.31 \pm 10.16$ & $98.16 \pm 10.85$ & 0.951 \\
\hline & $\mathrm{T} 1$ & $92.14 \pm 10.13$ & $93.36 \pm 10.32$ & 0.852 \\
\hline & $\mathrm{T} 2$ & $94.45 \pm 11.79$ & $93.34 \pm 102.6$ & 0.932 \\
\hline & T3 & $97.43 \pm 7.72$ & $96.99 \pm 5.51$ & 0.985 \\
\hline & $\mathrm{T} 4$ & $96.71 \pm 8.51$ & $95.83 \pm 8.2$ & 0.083 \\
\hline & $\mathrm{T} 1$ & $71.24 \pm 9.20$ & $72.41 \pm 10.79$ & 0.69 \\
\hline \multirow[t]{3}{*}{ HR (per minute) } & $\mathrm{T} 2$ & $100 \pm 7.85$ & $103.13 \pm 6.61$ & 0.107 \\
\hline & T3 & $74.67 \pm 9.67$ & $75.13 \pm 9.96$ & 0.855 \\
\hline & T4 & $75.6 \pm 8.99$ & $73.8 \pm 8.58$ & 0.431 \\
\hline \multirow[t]{5}{*}{ CVP $(\mathrm{mm} \mathrm{Hg})$} & T0 & $17.23 \pm 2.71$ & $17.33 \pm 2.4$ & 0.988 \\
\hline & $\mathrm{T} 1$ & $13.23 \pm 2.01$ & $13.10 \pm 1.79$ & 0.403 \\
\hline & $\mathrm{T} 2$ & $13.93 \pm 2.07$ & $14.33 \pm 1.75$ & 0.42 \\
\hline & T3 & $13.5 \pm 1.66$ & $13.43 \pm 1.78$ & 0.94 \\
\hline & $\mathrm{T} 4$ & $13.37 \pm 1.59$ & $13.10 \pm 1.47$ & 0.502 \\
\hline \multirow[t]{5}{*}{$\mathrm{Cl}\left(\mathrm{CL} / \mathrm{min} / \mathrm{m}^{2}\right)$} & T0 & $1.77 \pm 0.4$ & $1.83 \pm 0.4$ & 0.561 \\
\hline & $\mathrm{T} 1$ & $1.82 \pm 0.3$ & $1.84 \pm 0.41$ & 0.86 \\
\hline & $\mathrm{T} 2$ & $1.83 \pm 0.4$ & $1.91 \pm 0.37$ & 0.867 \\
\hline & T3 & $2.02 \pm 0.39$ & $2.08 \pm 0.39$ & 0.602 \\
\hline & $\mathrm{T} 4$ & $2.25 \pm 0.34$ & $2.22 \pm 0.38$ & 0.747 \\
\hline \multirow[t]{5}{*}{ PCWP $(\mathrm{mm} \mathrm{Hg})$} & T0 & $18.5 \pm 2.6$ & $18.47 \pm 2$ & 0.89 \\
\hline & $\mathrm{T} 1$ & $18.3 \pm 2.14$ & $18.42 \pm 1.97$ & 0.59 \\
\hline & $\mathrm{T} 2$ & $18.83 \pm 2.26$ & $18.57 \pm 1.98$ & 0.629 \\
\hline & T3 & $17.4 \pm 1.28$ & $12.37 \pm 1.92$ & 0.928 \\
\hline & $\mathrm{T} 4$ & $17.23 \pm 1.3$ & $17.17 \pm 137$ & 0.915 \\
\hline \multirow[t]{5}{*}{ SVRI (dyne/s/cm5/m²) } & T0 & $2144.43 \pm 230.08$ & $2195.53 \pm 238.94$ & 0.564 \\
\hline & $\mathrm{T} 1$ & $2060.72 \pm 224.46$ & $2110 \pm 236.16$ & 0.471 \\
\hline & $\mathrm{T} 2$ & $2079.83 \pm 228.46$ & $2131.63 \pm 237.79$ & 0.478 \\
\hline & T3 & $2024.6 \pm 223.49$ & $2074.7 \pm 242.29$ & 0.574 \\
\hline & $\mathrm{T} 4$ & $1967.9 \pm 223.7$ & $2016.1 \pm 242.57$ & 0.584 \\
\hline \multirow[t]{5}{*}{ PVRI (dyne/s/cm5/m²) } & T0 & $264.5 \pm 43.78$ & $272.4 \pm 50.96$ & 0.522 \\
\hline & $\mathrm{T} 1$ & $254.82 \pm 42.14$ & $264.78 \pm 49.21$ & 0.521 \\
\hline & $\mathrm{T} 2$ & $258.87 \pm 44.14$ & $266.77 \pm 51.27$ & 0.525 \\
\hline & T3 & $246.7 \pm 43.89$ & $251.1 \pm 43.65$ & 0.635 \\
\hline & T4 & $241.17 \pm 43.66$ & $246.63 \pm 43.95$ & 0.631 \\
\hline
\end{tabular}

Abbreviations: $\mathrm{Cl}$, cardiac index; CVP, central venous pressure; $\mathrm{HR}$, heart rate; MAP, mean arterial pressure; PCWP, pulmonary capillary wedge pressure; PVRI, pulmonary vascular resistance index; SVRI, systemic vascular resistance index. Note: $p$-Value $<0.05$ is considered statistically significant.

There are other proposed mechanisms promoting the anti-inflammatory properties of ketamine. Various studies reveal that ketamine favorably modulates the cellular immunity and neurohumoral response mechanism ${ }^{22}$ in conjunction to anti-analgesic effects. Low-dose ketamine during induction of general anesthesia influences cellular immunity as outlined by an attenuation of neutrophil superoxide production in CABG. ${ }^{14}$ Moreover, ketamine has also been shown to inhibit transcription factors NF-k $\beta$ and activator protein 1 (AP-1) in human blood neutrophils. ${ }^{23}$ These transcription factors regulate the transcription of genes encoding the production of proinflammatory cytokines such as IL-6, TNF- $\alpha$, IL-1, 
Table 3 Comparison of inflammatory markers between the control and test group

\begin{tabular}{|c|c|c|c|c|}
\hline Parameters & Time & Control group $(n=30)$ & Test group $(n=30)$ & $p$-Value \\
\hline \multirow[t]{5}{*}{ CRP (mg/dL) } & T0 & $1.97 \pm 1.08$ & $2.15 \pm 1.28$ & 0.684 \\
\hline & $\mathrm{T} 1$ & $72.81 \pm 6.24$ & $55.23 \pm 5.63$ & $<0.001$ \\
\hline & $\mathrm{T} 2$ & $73.282 \pm 6.36$ & $56.29 \pm 5.97$ & $<0.001$ \\
\hline & $\mathrm{T} 3$ & $80.86 \pm 6.43$ & $62.76 \pm 5.35$ & $<0.001$ \\
\hline & $\mathrm{T} 4$ & $86.11 \pm 7.18$ & $67.19 \pm 5.96$ & $<0.001$ \\
\hline \multirow[t]{5}{*}{ IL-6 (pg/mL) } & T0 & $22.06 \pm 3.71$ & $22.07 \pm 4.36$ & 0.987 \\
\hline & $\mathrm{T} 1$ & $110.23 \pm 8.52$ & $59.08 \pm 11.23$ & $<0.001$ \\
\hline & $\mathrm{T} 2$ & $113.56 \pm 8.94$ & $62.68 \pm 11.31$ & $<0.001$ \\
\hline & T3 & $133.93 \pm 10.83$ & $74.05 \pm 13.42$ & $<0.001$ \\
\hline & T4 & $131.54 \pm 22.7$ & $83.49 \pm 13.47$ & $<0.001$ \\
\hline \multirow[t]{5}{*}{ TNF- $\alpha(p g / m L)$} & T0 & $12.32 \pm 1.64$ & $12.01 \pm 1.66$ & 0.469 \\
\hline & $\mathrm{T} 1$ & $13.24 \pm 1.32$ & $13.16 \pm 1.46$ & 0.524 \\
\hline & $\mathrm{T} 2$ & $14.38 \pm 1.45$ & $14.16 \pm 1.56$ & 0.574 \\
\hline & T3 & $14.72 \pm 1.49$ & $14.63 \pm 1.30$ & 0.818 \\
\hline & T4 & $15.14 \pm 1.24$ & $5.23 \pm 1.25$ & 0.764 \\
\hline \multirow[t]{5}{*}{$\mathrm{TnT}(\mathrm{ng} / \mathrm{L})$} & T0 & $8.9 \pm 2.07$ & $9.2 \pm 2.16$ & 0.687 \\
\hline & $\mathrm{T} 1$ & $63.72 \pm 5.08$ & $61.47 \pm 5.57$ & 0.143 \\
\hline & $\mathrm{T} 2$ & $64.77 \pm 5.91$ & $62.57 \pm 5.75$ & 0.149 \\
\hline & $\mathrm{T3}$ & $117.4 \pm 6.04$ & $116.23 \pm 7.01$ & 0.619 \\
\hline & $\mathrm{T} 4$ & $123.52 \pm 4.61$ & $122.8 \pm 4.33$ & 0.509 \\
\hline \multirow[t]{5}{*}{ CK-MB (IU/L) } & T0 & $14.13 \pm 5.23$ & $14.17 \pm 4.98$ & 0.98 \\
\hline & $\mathrm{T} 1$ & $81.21 \pm 6.33$ & $82.63 \pm 6.97$ & 0.298 \\
\hline & $\mathrm{T} 2$ & $8.23 \pm 6.66$ & $83.73 \pm 7.07$ & 0.324 \\
\hline & T3 & $74.37 \pm 5.69$ & $74.77 \pm 6.17$ & 0.8 \\
\hline & $\mathrm{T} 4$ & $75.13 \pm 6.38$ & $74.23 \pm 5.62$ & 0.528 \\
\hline
\end{tabular}

Abbreviations: CK-MB, creatine kinase muscle brain; CRP, C-reactive protein; IL-6, interleukin 6; TNF- $\alpha$, tissue necrosis factor- $\alpha$; TnT, troponin T. Note: $p$-Value $<0.05$ is considered statistically significant.

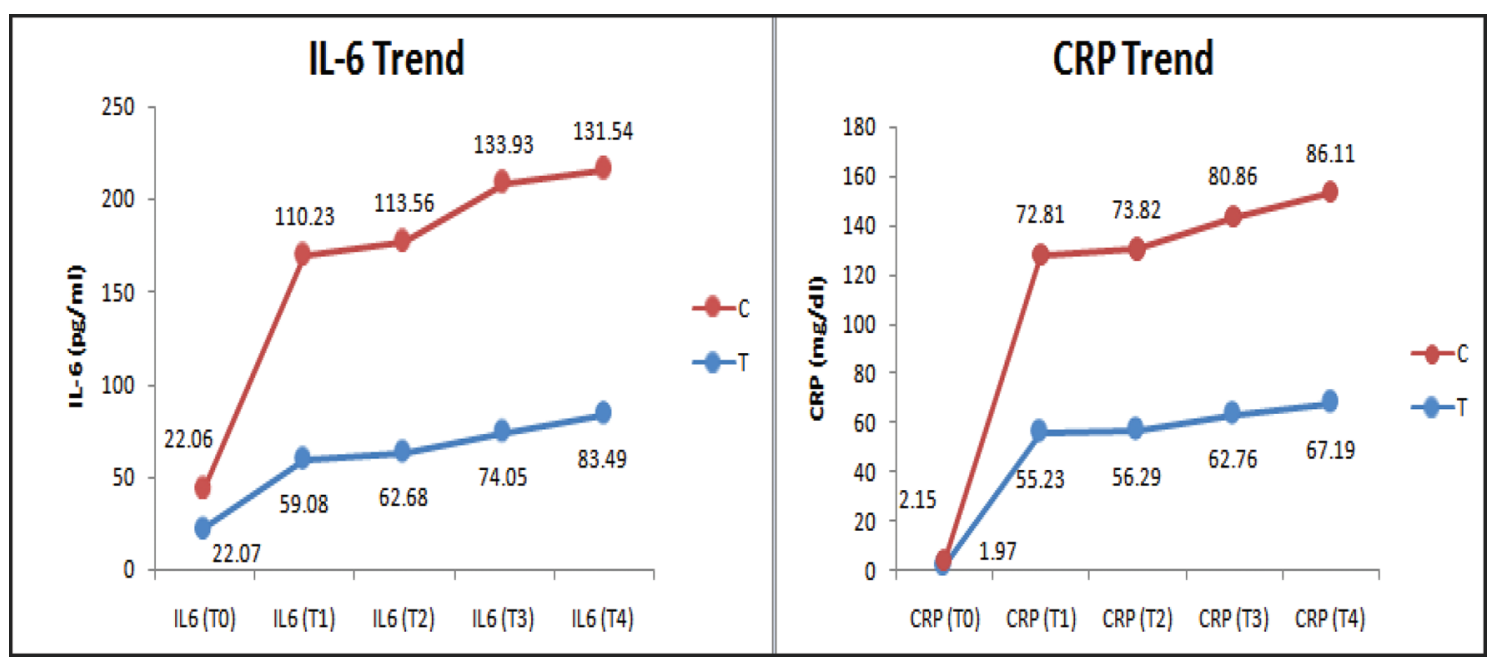

Fig. 1 Comparison of biochemical marker trends in the control and test group. 
Table 4 Comparison of outcomes between the control and test group

\begin{tabular}{|l|l|l|l|}
\hline Parameters & $\begin{array}{l}\text { Control group } \\
(\boldsymbol{n}=\mathbf{3 0})\end{array}$ & $\begin{array}{l}\text { Test group } \\
(\boldsymbol{n}=\mathbf{3 0})\end{array}$ & $p$-Value \\
\hline Duration of mechanical ventilation (h) & $16.36 \pm 3.84$ & $15.85 \pm 1.98$ & 0.336 \\
\hline Length of ICU stay (d) & $4.3 \pm 0.82$ & $4.23 \pm 0.56$ & 0.891 \\
\hline Length of hospital stay (d) & $7.42 \pm 1.48$ & $7.13 \pm 1.28$ & 0.823 \\
\hline Mortality (\%) & 1 & 1 & 1 \\
\hline $\mathrm{VIS}_{\mathrm{h}}$ & $0.52 \pm 0.08$ & $0.36 \pm 0.07$ & $<0.001$ \\
\hline
\end{tabular}

Abbreviations: ICU, intensive care unit; $\mathrm{VIS}_{H}$, highest postoperative vasopressor inotrope score. Note: $p$-Value $<0.05$ is considered statistically significant.

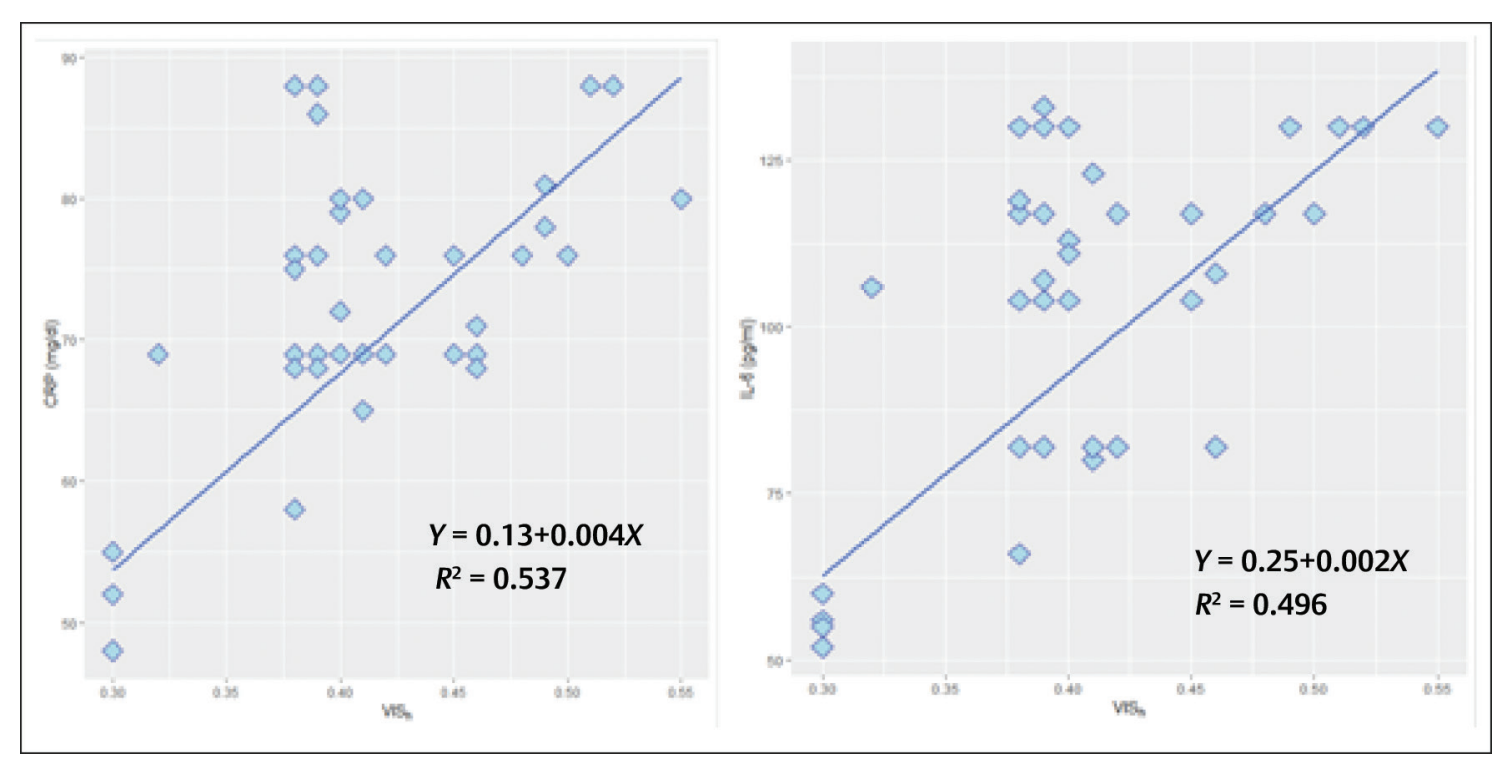

Fig. 2 The correlation plots of average postoperative CRP and IL- 6 with the highest postoperative vasopressor inotrope score (VIS $S_{H}$ ) CRP, C-reactive protein; IL-6, interleukin-6.

and IL-8. Therefore, inhibition of transcription factors, AP-1 and NF-k $\beta$ is a key mechanism by which ketamine attenuates the cytokine release and resultant inflammatory response observed in cardiac surgery.

Several caveats surface on a closer evaluation of this clinical area of recent interest, aimed at demonstrating anti-inflammatory effect of anesthetic agents like ketamine in cardiac surgical patients. First, the available literature elucidates that the anti-inflammatory effect of ketamine is dose-related ${ }^{24}$ and higher doses of ketamine can attenuate inflammatory reactions to a greater extent. However, owing to its sympathomimetic effects, ketamine can elicit adverse hemodynamic modifications toward an increase in myocardial oxygen consumption. ${ }^{25}$ Therefore, subanesthetic dose, as low as $0.5 \mathrm{mg} / \mathrm{kg}$ was employed in this study. Second, this low dose of ketamine did not result in untoward hemodynamic perturbations such as significant elevation in PVR. Moreover, all patients in the study received midazolam to prevent ketamine-induced hallucinations. Finally, the conduct of the study in a tertiary care center by a single experienced cardiac surgeon and adherence to standard institutional protocol constitute the inherent strength of the study.
The study had a few inherent limitations. The study did not assess anti-inflammatory mediator such as IL-10, other proinflammatory makers such as IL-8 and IL-2 and therefore could not assess any effects of ketamine on these mediators. Conflicting results have been reported regarding the ketamine's effect on the IL-10, and although low-dose ketamine was reported to attenuate increases in IL-10 in OPCAB. ${ }^{13}$ Other studies ${ }^{26}$ have shown significantly higher levels of IL-10 at the end of surgery. The other limitation of the study was the duration of follow-up after surgery which was kept as 48 hours. Studies ${ }^{17}$ have shown that inflammatory markers, namely IL-6 and CRP did not return to the baseline level even after 30 days postoperatively. This protracted period of activation of the inflammatory reaction parallels an increase in the risk of cardiovascular events in the recovery phase after the surgery.

\section{Conclusion}

The results of the study indicate that administration of low dose of ketamine prior to induction of anesthesia in patients undergoing OPCAB, induces a statistically significant 
attenuation in the production of proinflammatory mediators, IL-6 and CRP. No significant difference was observed in the levels of TNF- $\alpha, \mathrm{CK}-\mathrm{MB}$, TnT, and hemodynamic parameters between the ketamine and control group.

The findings of the study substantiate the anti-inflammatory potential of ketamine in preventing immune function alterations attributable to anesthesia and cardiac surgery.

\section{Conflict of Interest}

None declared.

\section{References}

1 Verma S, Fedak PW, Weisel RD, et al. Off-pump coronary artery bypass surgery: fundamentals for the clinical cardiologist. Circulation 2004;109(10):1206-1211

2 Bolli R, Becker L, Gross G, Mentzer R Jr, Balshaw D, Lathrop DA; NHLBI Working Group on the Translation of Therapies for Protecting the Heart from Ischemia. Myocardial protection at a crossroads: the need for translation into clinical therapy. Circ Res 2004;95(2):125-134

3 Cremer J, Martin M, Redl H, et al. Systemic inflammatory response syndrome after cardiac operations. Ann Thorac Surg 1996;61(6):1714-1720

4 Deng MC, Dasch B, Erren M, Möllhoff T, Scheld HH. Impact of left ventricular dysfunction on cytokines, hemodynamics, and outcome in bypass grafting. Ann Thorac Surg 1996;62(1):184-190

5 Hennein HA, Ebba H, Rodriguez JL, et al. Relationship of the proinflammatory cytokines to myocardial ischemia and dysfunction after uncomplicated coronary revascularization. J Thorac Cardiovasc Surg 1994;108(4):626-635

6 Hall RI, Smith MS, Rocker G. The systemic inflammatory response to cardiopulmonary bypass: pathophysiological, therapeutic, and pharmacological considerations. Anesth Analg 1997;85(4):766-782

7 Sun J, Wang XD, Liu H, Xu JG. Ketamine suppresses endotoxin-induced NF-kappaB activation and cytokines production in the intestine. Acta Anaesthesiol Scand 2004;48(3):317-321

8 Harig F, Hohenstein B, von der Emde J, Weyand M. Modulating IL-6 and IL-10 levels by pharmacologic strategies and the impact of different extracorporeal circulation parameters during cardiac surgery. Shock 2001;16(suppl 1) :33-38

9 Paparella D, Yau TM, Young E. Cardiopulmonary bypass induced inflammation: pathophysiology and treatment. An update. Eur J Cardiothorac Surg 2002;21(2):232-244

10 Asimakopoulos G, Gourlay T. A review of anti-inflammatory strategies in cardiac surgery. Perfusion 2003;18(suppl 1) :7-12

11 Goudeau JJ, Clermont G, Guillery O, et al. In high-risk patients, combination of antiinflammatory procedures during cardiopulmonary bypass can reduce incidences of inflammation and oxidative stress. J Cardiovasc Pharmacol 2007;49(1):39-45
12 Roytblat L, Roy-Shapira A, Greemberg L, et al. Preoperative low dose ketamine reduces serum interleukin-6 response after abdominal hysterectomy. Pain Clin 1996;9:327-334

13 Bartoc C, Frumento RJ, Jalbout M, Bennett-Guerrero E, Du E, Nishanian E. A randomized, double-blind, placebo-controlled study assessing the anti-inflammatory effects of ketamine in cardiac surgical patients. J Cardiothorac Vasc Anesth 2006;20(2):217-222

14 Zilberstein G, Levy R, Rachinsky M, et al. Ketamine attenuates neutrophil activation after cardiopulmonary bypass. Anesth Analg 2002;95(3):531-536

15 Roytblat L, Talmor D, Rachinsky M, et al. Ketamine attenuates the interleukin- 6 response after cardiopulmonary bypass. Anesth Analg 1998;87(2):266-271

16 Kanda T, Takahashi T. Interleukin-6 and cardiovascular diseases. Jpn Heart J 2004;45(2):183-193

17 Kawasaki C, Kawasaki T, Ogata M, Nandate K, Shigematsu A. Ketamine isomers suppress superantigen-induced proinflammatory cytokine production in human whole blood. Can J Anaesth 2001;48(8):819-823

18 Caputti GM, Palma JH, Gaia DF, Buffolo E. Off-pump coronary artery bypass surgery in selected patients is superior to the conventional approach for patients with severely depressed left ventricular function. Clinics (São Paulo) 2011;66(12):2049-2053

19 Loix S, De Kock M, Henin P. The anti-inflammatory effects of ketamine: state of the art. Acta Anaesthesiol Belg 2011;62(1):47-58

20 Cho JE, Shim JK, Choi YS, Kim DH, Hong SW, Kwak YL. Effect of low-dose ketamine on inflammatory response in offpump coronary artery bypass graft surgery. $\mathrm{Br} \mathrm{J}$ Anaesth 2009;102(1):23-28

21 Ruane-O'Hora T, Hall WJ, Markos F. The effect of ketamine and saffan on the beta-endorphin and ACTH response to haemorrhage in the minipig. Physiol Res 2008;19:276-282

22 Mazar J, Rogachev B, Shaked G, et al. Involvement of adenosine in the antiinflammatory action of ketamine. Anesthesiology 2005;102(6):1174-1181

23 Hanouz JL, Zhu L, Persehaye E, et al. Ketamine preconditions isolated human right atrial myocardium: roles of adenosine triphosphate-sensitive potassium channels and adrenoceptors. Anesthesiology 2005;102(6):1190-1196

24 Cook DJ, Housmans PR, Rorie DK. Effect of ketamine $\mathrm{HCl}$ on norepinephrine disposition in isolated ferret ventricular myocardium. J Pharmacol Exp Ther 1992;261(1):101-107

25 Gurfinkel R, Czeiger D, Douvdevani A, et al. Ketamine improves survival in burn injury followed by sepsis in rats. Anesth Analg 2006;103(2):396-402

26 Welters ID, Feurer MK, Preiss V, et al. Continuous S-(+)ketamine administration during elective coronary artery bypass graft surgery attenuates pro-inflammatory cytokine response during and after cardiopulmonary bypass. $\mathrm{Br} \mathrm{J}$ Anaesth 2011;106(2):172-179 